\title{
Electrocutaneous reflexes and multimodality evoked potentials in multiple sclerosis
}

\author{
W G Friedli, P Fuhr
}

\begin{abstract}
Electrical stimulation of the digital nerves of the index finger produces changes in the EMG signal during steady voluntary contraction of the first dorsal interosseous muscle. This electrocutaneous reflex (ECR) was studied in 90 patients classified into different categories according to diagnostic criteria for multiple sclerosis. In addition, pattern reversal visual evoked responses (VER), brainstem auditory evoked responses (BAER) as well as spinal and scalp recorded somatosensory evoked potentials (SSEP) were investigated by stimulating both the index finger and the posterior tibial nerve. The reflex response was altered uni- or bilaterally in 56 per cent of the patients and the abnormalities of the ECR were related to the categories of diagnostic probability. Pathological results were found in 28 per cent of the hands without clinical evidence of sensorimotor deficit. Although ECR abnormalities were often associated with clinical signs, significant correlation was found only with hyperreflexia and/or increased muscle tone in the corresponding upper limb. Pathological ECR were more common than pathological SSEPs with finger stimulation recorded under identical stimulus conditions. Fewer abnormalities were found by ECR testing than with VER, but the proportion of abnormal ECR was higher than that of BAER. ECR provides a valuable supplement to existing electrophysiological procedures for detecting lesions in the central nervous system.
\end{abstract}

Department of

Neurology, EMG

Laboratory,

University Hospital,

Basel, Switzerland

W G Friedli

P Fuhr*

$\star$ Present address:

National Institute of

Neurological and

Communicative

Disorders and Stroke,

National Institutes of

Health, Bethesda, MD,

United States.

Correspondence to:

Walter G Friedli,

Neurologische

Universitätsklinik,

Kantonsspital, CH-4031

Basel, Switzerland.

Received 10 April 1989 and in revised form 2 October

in revised 1989.

Accepted 30 October 1989

Changes of rectified and averaged EMG activity seen within the first $100 \mathrm{~ms}$ following the electrocutaneous stimulus are characterised by a triphasic modulation of activity. A shortlatency increase (E1) and a later positive peak (E2) are separated by a decrease in EMG activity (I1) while later changes of the signal following E2 are inconsistent. This electrocutaneous reflex (ECR) was investigated systematically by using a method similar to that presented by Jenner and Stephens in $1981 .^{1}$ Information on the range of normality and reliability of the ECR in adults was given in our previous paper. ${ }^{2}$ reflex response in upper limbs were described with lesions of the contralateral sensorimotor cortex, ${ }^{4-8}$ lesions of the ipsilateral dorsal columns and the corticospinal tract, ${ }^{15}$ whereas no alteration occurred in patients with lesions affecting the frontal lobe, occipital lobe, cerebellum or spinothalamic pathways. ${ }^{1}$

The aim of this study was to evaluate the ECR as a screening method in patients suffering from multiple sclerosis and to compare its diagnostic significance with established electrodiagnostic procedures. ${ }^{9-11}$

\section{Methods}

Electrophysiological studies were performed on 90 patients, 59 women and 31 men, ranging in age from 14 to 67 years (median 40 years), admitted to our laboratory on the suspicion of multiple sclerosis. Based on clinical and laboratory findings the patients were classified into diagnostic categories according to the criteria of Poser et al in $1983^{12}$ (table 1). In contrast to those criteria we required pathological IgG production and oligoclonal bands for laboratory support of diagnostic categories. Patients with marked paresis of the upper limb (MRC Grade 4 and below) who unable to perform a steady isometric contraction of the hand muscles were excluded.

In addition, magnetic resonance imaging (MRI) was performed on 37 patients. BAERs, and SSEPs were all investigated by stimulating both the index finger and the posterior tibial nerve for most patients.

\section{Electrocutaneous reflex}

The subjects, who were lying comfortably in a supine position, placed their hands with palm down on a metal plate on which a force transducer was mounted. Pressure against the load cell was provided by abduction of the index finger while the ulnar edge of the hand

There is evidence that the late component of the reflex response depends on the integrity of supraspinal structures. ${ }^{13-7}$ Changes of the was supported by another metal piece which was adjusted to the size of the hand. The ECR was recorded while the patients performed an isometric contraction of the first dorsal interosseous muscle at a standardised force which

Table 1 Diagnostic classification of the patient population

\begin{tabular}{ll}
\hline Probability of multiple sclerosis & $\begin{array}{l}\text { Number of } \\
\text { patients }\end{array}$ \\
\hline Clinically definite MS (CD) & $33(36 \cdot 7 \%)$ \\
Laboratory supported definite MS (LD) & $18(20 \cdot 0 \%)$ \\
Clinically probable MS (CP) & $33(36 \cdot 7 \%)$ \\
Laboratory supported probable MS (LP) & $6(6 \cdot 6 \%)$ \\
Total & $90(100 \%)$ \\
\hline
\end{tabular}


Figure 1 Normal configuration of the $E C R$ as recorded from the first dorsal interosseus muscle by electrical stimulation of the index finger. Peak latencies of $E 1, I 1$ and $E 2$ were measured and the amplitude-quotient ( $A Q$ as given by $A 1 / A 2$ was calculated. Data from one female subject of 22 years.

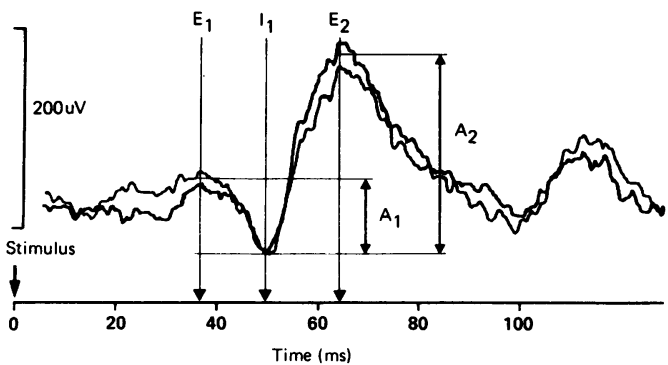

was $20 \%$ of maximum voluntary contraction. Force was kept constant with visual feedback by using an analogue scale voltmeter connected to the load cell. Rectangular current pulses of $0.2 \mathrm{~ms}$ were applied at a frequency of $3 / \mathrm{s}$ with ring electrodes, which were fixed proximally (cathode) and distally (anode) to the proximal interphalangeal joint of the index finger and connected to an isolated constant current stimulator (TOENNIES, DA II). Stimulus strength was standardised at four times the sensory threshold as assessed by the method of descending limits on both sides. Electrical activity was recorded from the first dorsal interosseous muscle with surface electrodes, the indifferent electrode being positioned over the processus styloideus radii. EMG activity was amplified by using a differential amplifier $(20 \mathrm{~Hz}$ to $1 \mathrm{kHz})$, rectified and averaged time locked to the stimulus. The sampling period was $200 \mathrm{~ms}$ and 512 samples were averaged twice on either side. Skin temperature was kept constant at $34^{\circ} \mathrm{C}$ by means of a temperature control unit (DISA, Type $15 \mathrm{H} \mathrm{02).} \mathrm{The} \mathrm{ECR}$ as characterised by a triphasic modulation of activity E1-I1-E2 (fig 1) was assessed as being within normal limits or not by using the upper limits of confidence proposed previously for healthy adults (table 2 ). ${ }^{2}$

\section{Pattern reversal visual evoked responses}

A checkerboard was projected by a mirror onto a translucent screen subtending $18^{\circ}$ of the patients visual field. The size of individual squares was $42 \mathrm{~min}$ of arc, the luminance of the white squares was $85 \mathrm{~cd} / \mathrm{m}^{2}$ and that of the black squares $2 \mathrm{~cd} / \mathrm{m}^{2}$. Pattern reversal rate was $0.5 \mathrm{~Hz}$. Monocular stimulation was performed while the patients fixated a red dot in the centre of the screen. The response was recorded from an active needle electrode at $\mathrm{Oz}$ with a reference at $\mathrm{Fz}$ (10-20-system) and amplified with a bandpass of $0.5-100 \mathrm{~Hz}$. Analysis time was 200 $\mathrm{ms}$ and 256 samples were averaged twice on either side. Absolute latency and interocular difference of the $P_{100}$ component were considered as abnormal if they exceeded the mean

Table 2 Upper limits of confidence (mean +2.5 SD) for absolute and adjusted ${ }^{\star}$ data (latencies in $\mathrm{ms}$ )

\begin{tabular}{lllll}
\hline & $\begin{array}{l}\text { Absolute } \\
\text { peak } \\
\text { latency }\end{array}$ & $\begin{array}{l}\text { Absolute } \\
\text { side } \\
\text { difference }\end{array}$ & $\begin{array}{l}\text { Adjusted } \\
\text { peak } \\
\text { latency }\end{array}$ & $\begin{array}{l}\text { Adjusted } \\
\text { side } \\
\text { difference }\end{array}$ \\
\hline E1 & 48.3 & $5 \cdot 1$ & 68.6 & 7.6 \\
11 & 59.7 & 4.4 & 84.8 & 6.6 \\
E2 & 80.3 & 10.9 & 117.4 & 15.9 \\
AQ (A1/A2) & 1.45 & & & \\
\hline
\end{tabular}

*Adjustment of arm length to a standard of $100 \mathrm{~cm}$. normal by more than 2.5 standard deviations (SD).

\section{Brainstem auditory evoked responses}

Stimuli consisted of unfiltered clicks of $0.15 \mathrm{~ms}$ duration and an intensity of $70 \mathrm{~dB}$ above the patient's hearing threshold, presented monaurally via earphones at a rate of $10 / \mathrm{s}$, while the unstimulated ear was masked with white noise at $70 \mathrm{~dB}$. The polarity of the click stimuli was reversed in regular intervals by giving condensation and rarefaction clicks in blocks of 512 samples within the same averaging run. Recording electrodes were set at $\mathrm{Cz}-\mathrm{Al}$ and $\mathrm{Cz}-$ A2 and connected to differential amplifiers $(150 \mathrm{~Hz}-3 \mathrm{kHz})$. The sampling period was 10 $\mathrm{ms}$ and 2048 samples were averaged twice for each side. The responses were regarded as abnormal if peak latencies and side difference of the components III and V (as measured from the stimulus artifact), and the I-V interval were beyond 2.5 SDs from the mean of the normal population.

\section{Somatosensory evoked responses with index finger stimulation}

Identical stimuli were used for recordings of both SSEP Dig II and ECR. SSEPs were recorded with stainless steel needle electrodes over the contralateral somatosensory hand area ( $3 \mathrm{~cm}$ behind $\mathrm{C} 3$ and $\mathrm{C} 4$ ) and over the cervical spinal cord at $\mathrm{C} 6 / \mathrm{C} 7$. The reference electrodes were placed on the midupper forehead $(\mathrm{Fz})$. The potentials were amplified using a bandpass of 0.5 to $3000 \mathrm{~Hz}$ and 512 samples were averaged twice for a sampling period of $50 \mathrm{~ms}$. Skin temperature was controlled and kept constant at $34^{\circ} \mathrm{C}$. Results were assessed as abnormal if the latency and the latency asymmetry of the cortical $\mathrm{N}_{22}$ exceeded the mean normal by more than 2.5 SDs for adjusted armlength, and if there was a reduction of the N22/P30 amplitude of more than 50 per cent compared to the contralateral side. Although recorded in all patients the cervical SSEPs were elicited with difficulty in some of them and did not allow unambiguous identification of individual components. Therefore, these responses were not taken into consideration.

Somatosensory evoked responses with stimulation of the posterior tibial nerve

Electrical stimuli of $0.2 \mathrm{~ms}$ duration were applied to the posterior tibial nerve at the ankle at a frequency of $3 / \mathrm{s}$. Stimulus intensity was adjusted to three times motor threshold according to twitches of plantar muscles of the foot. Evoked responses were recorded by needle electrodes at $\mathrm{Th} 12 / \mathrm{L} 1$ with a reference on the anterior-superior iliac spine and by steel needles applied to the scalp at $\mathrm{Cz}$ and Fpz. Signals were amplified $(0.5-3000 \mathrm{~Hz})$ and at least 512 samples were averaged twice on either side for a sampling period of $100 \mathrm{~ms}$. Skin temperature of the legs was kept constant at $34^{\circ} \mathrm{C}$. Provided that a normal spinal response was recorded, the latencies and the side-to-side differences of the cortical components $\mathbf{P}_{40}$ and $\mathrm{N}_{50}$ were considered as prolonged when their values exceeded the mean normal by more than 2.5 SDs. 
Figure 2 Examples of pathological ECR in two patients with clinically probable MS (CP): $A$ male patient of 19 years with abnormal ECR on the right hand as characterised by a small second peak ( $A Q$ beyond $1 \cdot 45$, that is, exceeding the upper limits of confidence) and a late excitatory component not seen on the left side; $B$ ) male patient of 38 years. Pathological reflex response on the left side not allowing identification of E2 (probably being delayed compared to the normal configuration of the ECR on the right).

Note the wide range of normality of the ECR with respect to its configuration ( $A Q)$ !
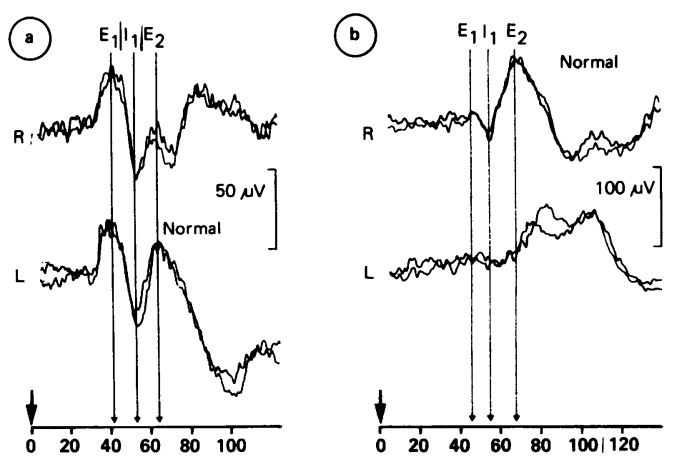

Results

Abnormalities of the ECR

In general, the normal pattern of the ECR to index stimulation consisted of two excitatory components (E1 and E2) separated by a decrease in EMG activity (I1) of the first dorsal interosseous muscle (fig 1). Since E1 is missed as a reliably identifiable component in about $10^{\circ}{ }^{\circ}$ of the normal subjects, ${ }^{2} \mathrm{E} 1-\mathrm{I} 1-\mathrm{E} 2$ and I1E2 were considered as normal, E1-I1 and a monophasic response as abnormal configurations. Later changes of the EMG signal following $E 2$ proved to be inconsistent and were not analysed therefore. Latencies were measured to the peaks of the reproducible maxima or minima of the reflex response. Based on our previous investigation in healthy adults ${ }^{2}$ the mean (SD) latency was 40.3 (3.2), 50.9 (3.5) and $65.8(5.8) \mathrm{ms}$ for E1, I1 and E2 respectively.

There was a strong linear correlation between the latencies and arm length whereas age and sex were of minor influence on the reflex response. Arm length as the most important factor of interindividual variability was eliminated by normalising the absolute latencies for an arm length of $100 \mathrm{~cm}$. The amplitudes as measured peak-to-peak between E1 and I1 (A1) as well as I1 and E2 (A2) were used for calculating the amplitude quotient A1/A2 (AQ). A1 was found to be smaller than A2 $(A Q<1.0)$ in $75 \%$ of normal subjects, whereas in $25 \% \mathrm{~A} 1$ was equal or larger than $\mathrm{A} 2$ $(\mathrm{AQ}=\mid>1.0)$ on one or both sides. Given a normal configuration of the ECR, the latter was assessed as abnormal if the latencies or the side differences of the various components exceeded the upper limits of confidence for adjusted arm length (table 2).

According to the criteria mentioned above 50 out of 90 patients $(56 \%)$ had ECR abnormalities. Alterations of the reflex responses

Table 3 Incidence of ECR abnormalities

\begin{tabular}{lcr}
\hline ECR findings & $\begin{array}{c}\text { Upper } \\
\text { limbs }\end{array}$ & \multicolumn{1}{c}{$\%$} \\
\hline Latency & 20 & $11 \cdot 1$ \\
E1 and I1 normal, E2 delayed & 28 & $15 \cdot 5$ \\
E1 normal, I1 and E2 delayed & & \\
Configuration & 2 & $1 \cdot 1$ \\
E1-I1, no E2 & 9 & 5.0 \\
E1, no I1, no E2 & 3 & $1 \cdot 7$ \\
Amplitude quotient A1/A2 $>1.45$ & 7 & 3.9 \\
Late monophasic response & 3 & $1 \cdot 7$ \\
No reproducible response & 108 & 60.0 \\
Normal response & 180 & $100 \cdot 0$ \\
Total & & \\
\hline
\end{tabular}

were found bilaterally in 22 patients whereas in 28 the investigation revealed pathological results in one hand (table 3). E1 being within normal limits, the latency of E2 was increased in 48 upper limbs ( $67 \%$ of pathological results), 36 of whom showed an increase of the E1-E2 interpeak-latency as well. Both I1 and E2 were delayed in 28 upper limbs. While the latencies proved to be the most sensitive parameters in our population, the configuration of the reflex response was decisive for ECR pathology in 24 upper limbs (33\% of pathological results). There was no reproducible E2 in 11 patients, in nine of whom both $\mathrm{I} 1$ and E2 were missed as reliably identifiable components. The amplitude quotient A1/A2 being decisive in only three patients, a late monophasic response (which could not be assigned to a specific component) was seen in seven upper limbs, while in another three a reproducible reflex response was not recorded at all. Two examples of pathological configuration of the ECR are given in fig 2 .

The frequency of ECR abnormalities increased in parallel with the probability of demyelinating disease. The reflex response was altered uni- or bilaterally in 34 out of 51 patients with definite MS $(67 \%)$ and in 16 of 33 patients assessed as having clinically probable MS $(48.5 \%)$. On the other hand, the ECR was normal in six patients with laboratory supported probable MS, so that the incidence of pathological findings was $41 \%$ in the sum total of patients with probable MS.

\section{ECR and multimodality evoked potentials}

Evoked potentials data are summarised in tables 4 and 5. VERs were recorded in 89 patients and found to be abnormal on one or both sides in 69 of them $(78 \%)$. SSEPs to finger stimulation recorded in 88 patients revealed a rather low incidence of pathological results $(28 \%)$ while abnormalities were found on one or both sides in $\mathbf{4 0}$ out of 78 patients examined with stimulation of the posterior tibial nerve $(51 \%)$. BAERs were studied in 86 patients; they were abnormal on one or both sides in 24 $(28 \%)$ and normal on both sides in 62 patients. Abnormalities of BAERs were found in a higher proportion of patients with clinically detectable brainstem lesion $(34 \%)$ while the incidence of pathological results was lower in the absence of corresponding signs $(24 \%)$. For all modalities tested the evoked potentials were found to be abnormal in a higher proportion of patients with definite MS (table 5).

The incidence 'of pathological ECR was higher than that of the somatosensory evoked potentials with cutaneous nerve stimulation in the upper limbs as recorded under identical stimulus conditions. Pathological reflex responses wère found in 40 hands without any abnormality of the SSEPs with finger stimulation. In 30 hands the examination of both ECR and SSEPs provided pathological results. From this group 15 had a prolonged latency and four a significantly longer latency of the N22 component compared to the contralateral side. In six hands index stimulation did not provide a reproducible cortical response while 
Table 4 Findings of multimodality evoked potentials

\begin{tabular}{llll}
\hline Modality & $\begin{array}{l}\text { Unilaterally } \\
\text { pathological }\end{array}$ & $\begin{array}{l}\text { Bilaterally } \\
\text { pathological }\end{array}$ & $\begin{array}{l}\text { Proportion of } \\
\text { pathological results }\end{array}$ \\
\hline VER & 24 & 45 & $69 / 89(78 \%)$ \\
SSEP tibial n & 14 & 26 & $40 / 78(51 \%)$ \\
SSEP index & 12 & 13 & $25 / 88(28 \%)$ \\
BAER & 13 & 11 & $24 / 86(28 \%)$ \\
ECR & 28 & 22 & $50 / 90(56 \%)$ \\
\hline
\end{tabular}

Table 5 Proportion of pathological results and probability of multiple sclerosis

\begin{tabular}{llr}
\hline Modality & $\begin{array}{l}\text { Definite } M S \\
(C D+L D)\end{array}$ & $\begin{array}{c}\text { Probable } M S \\
(C P+L P)\end{array}$ \\
\hline VER & $44 / 50(88 \cdot 0 \%)$ & $25 / 39(64 \cdot 1 \%)$ \\
SSEP tribial n & $23 / 44(52 \cdot 3 \%)$ & $17 / 34(50 \cdot 0 \%)$ \\
SSEP index & $18 / 50(36.0 \%)$ & $7 / 38(18 \cdot 4 \%)$ \\
BAER & $16 / 47(34 \cdot 0 \%)$ & $8 / 39(20 \cdot 5 \%)$ \\
ECR & $34 / 51(66 \cdot 7 \%)$ & $16 / 39(41 \cdot 0 \%)$ \\
\hline
\end{tabular}

Table 6 Pathological results without clinically evident sensory motor deficit

\begin{tabular}{llcc}
\hline & Pathol ECR & Normal ECR & Total hands \\
\hline Pathol SSEP & 12 & 7 & 19 \\
Normal SSEP & 24 & 87 & 111 \\
Total hands & 36 & 94 & 130 \\
\hline
\end{tabular}

the latter showed a significant amplitude reduction of more than 50 per cent side-toside-difference in four hands. Abnormal SSEPs over the somatosensory hand area were found in six patients (eight hands) in whom a reproducible ECR was recorded with stimulation of the corresponding index finger. $\mathrm{Al}$ though the reflex response was rather small in three of these cases, it was assessed as normal according to both latencies and configuration. In two other patients (two hands) with a delayed cortical response to index stimulation there was a striking configuration of the ECR as characterised by later components following the rather small E2. Nevertheless, these findings were assessed as being within normal limits according to the criteria mentioned above. There was a significant correlation between abnormal ECR and SSEP findings with stimulation of the corresponding index finger ( $p<0.001)$ for both the sum total of patients and those without sensorimotor deficit (table 6).

To evaluate the practical value of the ECR as a screening method in patients suspected of having MS, the incidence of pathological findings was compared to that of the multimodality evoked potentials. Abnormalities of the ECR were found in none of the eight patients without any pathological result provided by the well established electrophysiological screening tests. Positive findings for one modality were combined with one pathological reflex response in 17 out of 36 patients. Fourteen of 17 patients in the more certain diagnostic categories with positive results for at least three modalities also had alterations of the ECR.

ECR and clinical symptoms

Based on the clinical examination 38 of the 90 patients examined $(42 \%)$ showed signs of corticospinal lesion and/or sensory deficit

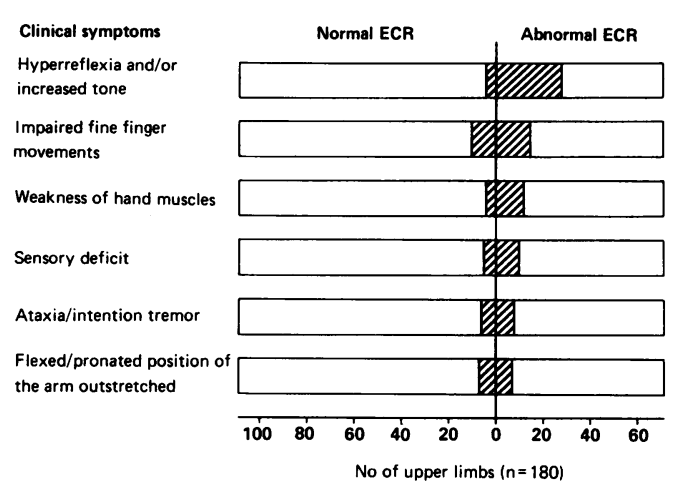

Figure 3 Correlation between abnormal

electrocutaneous reflex (ECR) and clinical symptoms in the corresponding upper limbs. By using the Chi square test, the proportion of limbs with a symptom and

abnormal ECR was compared to that of the limbs with the corresponding symptom and normal $E C R$. The categories of normal/abnormal ECR are separated by the vertical bar. "Hyperreflexia and/or increased muscle tone" as present in 32 upper limbs (hatched area) was the only symptom being significantly correlated with pathological $E C R(p<0.005)$.

unilaterally ( 26 patients) or in both upper limbs (12 patients). There was no evidence of a sensory or motor deficit in 130 of the 180 upper limbs examined $(72 \%)$. Abnormalities of the ECR were found in 36 hands (28\%) without clinical symptoms. From this group, 12 presented pathological SSEPs with finger stimulation (table 6 ). The relationship between clinical symptoms and ECR findings are summarised in fig 3. An abnormal reflex response was found in 36 out of 50 upper limbs $(72 \%)$ with signs of corticospinal lesion and/or sensory deficit whereas the ECR was within normal limits in 14 hands $(28 \%)$ in spite of clinical symptoms. The most frequent physical symptoms in our population were increased muscle tone and hyperreflexia in 32 upper limbs, 28 of whom showed an abnormal ECR (87\%). Impaired fine finger movements were seen in 25 hands, the ECR being abnormal in 15 of these $(60 \%)$. There was weakness of the hand muscles (including the first dorsal interosseous) in 16 upper limbs, 12 of which had an abnormal reflex response $(75 \%)$. If sensory testing was not performed by one of us, sensory deficits as described in the patients' records have been estimated cautiously and symptoms such as "numbness" and doubtful paresthesiae or dysesthesiae were not assessed as positive findings. Superficial and/or proprioceptive sensation were found to be impaired in 15 upper limbs, 10 of which presented alterations of the ECR as well as pathological SSEPs with stimulation of the index finger. Lack of position sense and abnormalities of postural sensation without other clinical deficits were found in three hands in which our investigations revealed abnormalities of both ECR and SSEPs with finger stimulation. Alternatively, symptoms such as ataxia, dysmetria and cerebellar tremor did not affect the frequency of pathological results.

In general, the ECR was more likely to be abnormal in upper limbs with sensory motor deficits. However, a significant correlation with pathological ECR was found only for hyperreflexia/increased muscle tone 
( $\mathrm{p}<0.005)$, which was the only sign associated with alterations of the ECR in eight hands. None of the other symptoms was significantly correlated with abnormal ECR. On the other hand, pathological findings were seen in a smaller proportion of hands in the absence of clinical symptoms and therefore provide evidence for subclinical lesions. Symptoms of past or actual brainstem lesions such as cranial nerve disorders, dizziness, nystagmus and diplopia were present in 34 of the 90 patients examined $(38 \%)$. ECR abnormalities were found uni- or bilaterally in $41 \%$ of the latter whereas 36 of 56 patients without corresponding signs $(64 \%)$ did present a pathological reflex response.

\section{Discussion}

This study was performed to evaluate the diagnostic significance of the ECR in 90 patients with probable or definite MS. We are aware of only one report on the application of electrically elicited long-latency reflexes in the upper limbs in MS with stimulation of the median as well as of the superficial radial nerve. ${ }^{13}$ To avoid interference from the muscle innervation we applied electrical stimuli to the index finger for the investigation of both ECR and somatosensory evoked potentials.

It is not known whether the long-latency components of the reflexes elicited through cutaneous excitation, muscle stretch and mixed nerve stimulation share a common pathway. Although a supraspinal origin of stretchreflexes is still controversial ${ }^{14-19}$ the dependence of the second EMG response on the integrity of both the central somatosensory afferent and the corticospinal pathways is suggested by previous results. ${ }^{20-22}$ Similar evidence was found for the long-latency reflex after mixed nerve ${ }^{48}$ and cutaneous stimulation. ${ }^{15}$ However, whether the ECR represents a transcortical or a spinal polysynaptic reflex loop that might be under suprasegmental modulating influence is unimportant for practical diagnostic purposes as long as this test discriminates reasonably well between the normal group and patients with MS.

ECR abnormalities were found in $\mathbf{5 0}$ of the 90 patients examined $(56 \%)$. The latency of the E2 component proved to be the most sensitive parameter, being responsible for $67 \%$ of pathological results. Abnormal configuration was decisive for pathology of the ECR in 24 upper limbs, three of whom did not show a reproducible reflex response. Validity of the ECR is provided by the high proportion of positive results in patients with clinically definite MS $(67 \%)$, that is, by an increasing incidence of pathological findings in parallel with the severity of clinical symptoms. On the other hand, an abnormal reflex response is obtained in $41 \%$ of the patients with probable MS and therefore ECR testing fulfils the requirements of a diagnostic test as needed in the less certain diagnostic categories.

Based on our results, subclinical lesions can be demonstrated in 36 of the 130 upper limbs without clinical evidence of corticospinal lesion or sensory deficit. However, the rather high number of neurologically intact arms $(58 \%$ of the patients examined) may be due in part to the fact that most of the clinical data had to be obtained from medical records which must imply some loss of information. Although clinical symptoms in the upper limbs are often associated with ECR abnormalities ( $72 \%$ ), the latter are found to be significantly correlated only with hyperreflexia and/or increased muscle tone in the corresponding arm. ECR testing has provided positive results in seven out of eight patients presenting a tetraspastic syndrome. Although the origin and the central pathways mediating the long-latency reflex response are not known definitively, abnormalities of the ECR should only be accepted as of diagnostic value in the sense of indicating subclinical lesions when there are no clinical symptoms of brainstem or spinal cord disease. ECR abnormalities were found in 36 of 56 patients without evidence of past or actual brainstem lesion. However, in only 16 of these (that is, in $29 \%$ of the population without brainstem lesion) there was no sensory motor deficit in the corresponding upper limb.

Although abnormal ECR is significantly correlated with abnormal SSEPs as recorded under identical stimulus conditions, ECR testing reveals a higher incidence of pathological results. Based on the hypothesis that the afferent pathway of the SSEPs is similar to or identical with that of the long-latency reflex response, an abnormal ECR is expected to occur in case of pathological SSEPs. On the other hand, the combination of pathological ECR with normal SSEPs (found in 40 hands in our study) suggests a lesion within the descending pathways mediating the reflex response. The findings in six patients (6 hands) exhibiting abnormal SSEPs without alterations of the ECR have to be emphasised and may be due to various factors. First, they might represent an argument for ECR and SSEPs to depend on different afferent somatosensory pathways, for example, the spinothalamic tract and the lemniscal afferent pathway which may be differentially affected by localised demylinating lesions. However, correlation of abnormality in these tests as found for the majority of patients exhibiting pathological SSEPs suggests their dependence on a common afferent pathway, provided that these findings do not represent two separate lesions. Second, the normal ECRs assessed by using the upper limits of confidence for certain parameters (not taking into account the absolute magnitude of the signal) might represent false negative results. By applying the procedure proposed previously for assessing the reflex response, false positive results are expected to occur in only about $0.5 \%$. Third, supposing a supraspinal reflex arc, the results may be influenced by the length of the pathways involved: SSEPs to finger stimulation may be impaired due to a lesion within the lemniscal pathway whereas the latter might have less influence proportionately on the ECR mediated by both ascending and descending pathways including synaptic relays. 
Fewer abnormalities are found by ECR testing than with VER, whereas the proportion of pathological results is higher for ECR than for both BAER and SSEPs. However, the evoked potentials might have been decisive as "paraclinical" evidence ${ }^{12}$ of a lesion in some patients and thus for inclusion in this study. By taking MRI findings as paraclinical evidence of lesions into consideration, electrophysiological results have been decisive for classification in 10 of the 33 patients with clinically probable MS (that is, in $30 \%$ of this group) while eight of the 33 patients $(24 \%)$ diagnosed as having clinically definite MS were identified by evoked potentials. Despite these difficulties a comparison of the diagnostic yield of the evoked responses with the results of the ECR might be of some interest for the following reasons. First, the bias resulting from this leads to an underestimation of the sensitivity of the ECR relative to the evoked potentials. Second, the findings of the established electrophysiological methods reported in this study are consistent with previous reports on the incidence of pathological results in MS. This suggests that the techniques used are comparable to those in earlier reports. In accordance with previous results, ${ }^{24}$ VERs most often led to the reclassification of patients and were decisive for the diagnosis of clinically probable and definite MS in seven patients $(11 \%)$ while in another eight patients $(12 \%)$ both VERs and SSEPs with tibial nerve stimulation revealed positive results. Reclassification was based on abnormal BAERs in only four of the 66 patients with clinically probable or definite MS $(6 \%)$.

Our data confirm previous reports on the incidence of the pathological VERs in MS, being lower in the less certain diagnostic categories. Abnormal VERs are found in 69 of the 89 patients examined $(78 \%), 19$ of whom with clinical evidence (4) or a history of optic neuritis (15). VER testing provides pathological results in 21 of 39 patients $(54 \%)$ in the category of probable MS without evidence of optic neuritis actually or in the past.

Dysfunction of the auditory pathways in the brain stem is shown in 24 of 86 patients which represents a rather low incidence of pathological results $(28 \%)$ compared with other reports. The proportion of BAER abnormalities in patients in the less diagnostic categories is only $20.5 \%$ in our study compared with $34.0 \%$ in patients with definite MS. Although we find a lower proportion of abnormalities in all categories of MS, the correlation between clinically detectable brainstem lesions and pathological BAERs is confirmed by our data. Abnormalities are obtained in $34 \%$ of MS patients with symptoms of brainstem lesion and in $24 \%$ of those without corresponding signs which is similar to previous results.

The rather low incidence of SSEP abnormalities with stimulation of the index finger $(28 \%)$ might be due to the small number of fibres involved compared to stimulation of the median nerve and also to the fact that we have not made diagnostic use of the cervical spinal SSEPs in spite of their diagnostic value in $M S .^{26}$ With stimulation of the posterior tibial nerve the incidence of abnormal SSEPs almost doubles that obtained by cutaneous nerve stimulation in the upper limbs. These findings may indicate impaired conduction in the dorsal columns of the spinal cord. SSEP abnormalities with tibial nerve stimulation are found in $23(52.3 \%)$ of the 44 patients examined with definite MS. However, positive results are of diagnostic value in only 15 of these $(34 \cdot 1 \%)$ without evidence of either brainstem or spinal cord disease. Similarly, abnormal SSEPs with tibial nerve stimulation in $17(50.0 \%)$ of the patients with probable $M S$ are indicative of an additional central nervous lesion in only 12 of them $(35 \cdot 3 \%)$.

ECR testing reveals a somewhat lower incidence of pathological results in our population than that provided by a previous study ${ }^{13}$ using mixed nerve stimulation in the upper limbs, but without taking into consideration clinical symptoms of the corresponding arm. Nevertheless, our findings also suggest that ECR testing is at least as effective as a diagnostic screening method in MS as the well established electrophysiological procedures, with the exception of the VERs. The ECR represents a valid supplement to the clinical examination for detection of lesions in the central nervous system. Moreover, since the latencies of the reflex response proved to be quite resistent to changes of the force of isometric contraction, ${ }^{2}$ ECR testing is readily available in any well equipped laboratory and therefore of practical value.

This work has been supported by The Schweizerische Multiple Sklerose Gesellschaft and by The CIBA Foundation. We thank Dr Mark Hallett and Dr Udo Kischka for their help.

1 Jenner JR, Stephens JA. Cutaneous reflex responses and their central nervous pathways studied in man. $J$ Physiol (Lond) 1982;333:405-19.

2 Fuhr P, Friedli WG. Electrocutaneous reflexes in upper limbs-reliability and normal values in adults. Eur Neurol 1987:-27:231-8.

3 Choa BHG, Stephens JA. Cutaneous reflex responses and central nervous lesions in man. Electroenceph Clin Neurophysiol 1981;52:S2

4 Conrad B, Aschoff JC. Effects of voluntary isometric and isotonic activity on late transcortical reflex components in normal subjects and hemiparetic patients. Electroenceph Clin Neurophysiol 1977;42:107-16.

5 Jenner JR, Stephens JA. Evidence for a transcortical cutaneous reflex response in man. $J$ Physiol (Lond) 1979;293:39P-40P

6 Rowlandson PH, Stephens JA. Cutaneous reflex responses recorded in children with various neurological disorders. Devl Med Child Neur 1985;27:434-47.

7 Deuschl G, Schenk E, Lücking $\mathrm{CH}$. Electrically elicited long-latency reflexes in thenar muscles: abnormal patterns
in central movement disorders. J Neurol (Suppl), in central m.

8 Claus D, Lang C, Kotzian J. Zur Beziehung zwischen Longloop Reflexbefund und Topographie von Hirninfarkten. ZEEG-EMG 1985;16:196-200.

9 Mills KR, Murray NMF. Corticospinal tract conduction time in multiple sclerosis. Ann Neurol 1985;18:601-5.

10 Hess CW, Mills KR, Murray NMF, Schriefer TN. Magnetic brain stimulation: central motor conduction studies in multiple sclerosis. Ann Neurol 1987;22:744-52.

11 Ingram DA, Thompson AJ, Swash M. Central motor conduction in multiple sclerosis: evaluation of abnormalities revealed by transcutaneous magnetic stimulation of the brain. J Neurol Neurosurg Psychiatry 1988;51: 487-94.

12 Poser CM, Paty DW, Scheinberg I, et al. New diagnostic criteria for multiple sclerosis: guidelines for research criteria for multiple sclerosis: guidelin

13 Deuschl D, Strahl K, Schenck E, Lücking $\mathrm{CH}$. The diagnostic significance of long-latency reflexes in multiple diagnostic significance of long-latency reflexes in multiple
sclerosis. Electroenceph clin Neurophysiol 1988;70:56-61.

14 Darton K, Lippold OCJ, Shahani M, Shahani U. Longlatency spinal reflexes in humans. $J$ Neurophysiol
1985;53:1604-18. 
15 Marsden CD, Merton PA, Morton HB. Servo action and stretch reflex in human muscle and its apparent dependence on peripheral sensation. $J$ Physiol (Lond) ence on peripher

16 Wiesendanger $M$, Miles TS. Ascending pathway of lowthreshold muscle afferents to the cerebral cortex and its possible role in motor control. Physiol Rev 1982;62: 1234-70

17 Matthews PBC. Evidence from the use of vibration that the human long latency stretch reflex depends upon spindle secondary afferents. J Physiol (Lond) 1984;348:383-415.

18 Hagbarth KE, Hagglund J, Wallin V, Young RR. Grouped spindle and electromyographic responses to abrupt wrist movements in man. J Physiol (Lond) $1981 ; 312 \cdot 81-96$.

19 Berardelli A, Hallett M, Kaufmann C, Fine E, Berenberg W, Simon SR. Stretch reflexes of triceps surae in normal man. J Neurol Neurosurg Psychiatry 1982;45:513-25.

20 Noth J, Podoll K, Friedemann HH. Long-loopn reflexes in small hand muscles studied in normal subjects and in patients with Huntington's disease. Brain 1985;108: patients
21 Marsden CD, Merton PA, Morton HB. Is the human stretch reflex cortical rather than spinal? Lancet 1973;i:759-61.

22 Marsden CD, Merton PA, Morton HB, Adam J. The effect of posterior column lesions on servo responses from the human

23 Marsden CD, Merton PA, Morton HB, Adam J. The role of the sensorimotor cortex and the capsular pathways on servo responses from the human long thumb flexor. Brain 1977;100:503-26.

$24 \mathrm{Kjaer} M$. The value of a multimodal evoked potential approach in the diagnosis of multiple sclerosis. In: Courjon J, Mauguire F, Revol M, eds. Clinical applications of evoked potentials in neurology. New York: Raven Press, 1982:507-12.

25 Chiappa KH, Harrison JL, Brooks EB, Young RR. Brainstem auditory evoked responses in 200 patients with multiple sclerosis. Ann Neurol 1980;7:135-43.

26 Small DG, Matthews WB, Small M. The cervical somatosensory evoked potentials in the diagnosis of multiple sclerosis. J Neurol Sci 1978;36:147-56. 